\title{
Author Correction: Human and mouse single-nucleus transcriptomics reveal TREM2-dependent and TREM2-independent cellular responses in Alzheimer's disease
}

Yingyue Zhou (D), Wilbur M. Song, Prabhakar S. Andhey, Amanda Swain (D), Tyler Levy, Kelly R. Miller, Pietro L. Poliani, Manuela Cominelli, Shikha Grover, Susan Gilfillan, Marina Cella, Tyler K. Ulland, Konstantin Zaitsev, Akinori Miyashita D, Takeshi Ikeuchi Di, Makoto Sainouchi, Akiyoshi Kakita, David A. Bennett, Julie A. Schneider, Michael R. Nichols, Sean A. Beausoleil D, Jason D. Ulrich, David M. Holtzman, Maxim N. Artyomov (D) and Marco Colonna (D)

Correction to: Nature Medicine https://doi.org/10.1038/s41591-019-0695-9, published online 13 January 2020.

In the version of this article initially published, the 'Data availability' statement indicated that the data had been deposited to Synapse; however, the data have since been relocated to the AD Knowledge Portal under study snRNAseqAD_TREM2. The statement has been updated to reflect this relocation and to include revised information on requests for data. The errors have been corrected in the HTML and PDF versions of the article.

Published online: 14 May 2020

https://doi.org/10.1038/s41591-020-0922-4

(c) The Author(s), under exclusive licence to Springer Nature America, Inc. 2020

\section{Author Correction: Respiratory virus shedding in exhaled breath and efficacy of face masks}

Nancy H. L. Leung (D, Daniel K. W. Chu, Eunice Y. C. Shiu, Kwok-Hung Chan, James J. McDevitt, Benien J. P. Hau, Hui-Ling Yen (D), Yuguo Li, Dennis K. M. Ip, J. S. Malik Peiris, Wing-Hong Seto, Gabriel M. Leung, Donald K. Milton and Benjamin J. Cowling (D)

Correction to: Nature Medicine https://doi.org/10.1038/s41591-020-0843-2, published online 3 April 2020.

In the version of this article initially published, in the first sentence of the third paragraph of the discussion, "for rhinovirus we detected virus in aerosols in 19 of 34 (56\%) participants (compared to 4 of 10 (40\%) for influenza and 8 of 23 (35\%) for coronavirus)" should have read "for rhinovirus we detected virus in aerosols in 19 of 34 (56\%) participants (compared to 4 of 10 (40\%) for coronavirus and 8 of $23(35 \%)$ for influenza)," with 'influenza' and 'coronavirus' transposed. The error has been corrected in the HTML and PDF versions of the article.

Published online: 27 May 2020

https://doi.org/10.1038/s41591-020-0946-9

(c) The Author(s), under exclusive licence to Springer Nature America, Inc. 2020 